\title{
Marker Quality Improvement in Augmented Reality Using Grey-scale Method
}

\author{
Dahliyusmanto* \\ Electrical Engineering Department \\ Universitas Riau \\ Indonesia
}

Eddy Hamdani

Electrical Engineering Department

Universitas Riau

Indonesia

\author{
Mohd Nizam Omar \\ InterNetWorks Lab \\ Universiti Utara Malaysia \\ Malaysia
}

Budhi Anto

Electrical Engineering Department

Universitas Riau

Indonesia

\author{
Devi Willieam Anggara \\ Electrical Engineering Department \\ Universitas Riau \\ Indonesia
}

\author{
Zuli Laili Isnaini \\ Japanese Languages and Education \\ Universitas Riau \\ Indonesia
}

*corresponding author: dahliyusmanto, dahliyusmanto@lecturer.unri.ac.id

\begin{abstract}
Augmented Reality (AR) is a technology which combines the real world and virtual world, where users can feel the interaction between the two worlds. Marker Augmented reality or known as marker-based tracking technique uses a marker as media to display this technology. The marker is used as an object that contains images with unique patterns to identify each different object. Because it has a unique pattern, not any objects can be used as a marker. In Vuforia SDK, image recognition is done using a grayscale technique to search for angular features of the pixel image. If the image was taken directly using the camera in the old times in the form of Black and White (BNW), it has bad quality and most likely cannot be used as a marker because it has a few features. In BNW images taken directly from the camera, the Vuforia SDK detects only a few features in the image. This journal explains how the technique of making a BNW image into a marker can be detected by Augmented Reality Technology.
\end{abstract}

Keywords- augmented reality, marker, vuforia SDK, greyscale.

\section{INTRODUCTION}

Augmented Reality is a technology that draws attention from all sorts of fields, whether in entertainment field such as games, healthcare, a field such as Magnetic Resonance Imaging (MRI), marketing field such as advertising and military field such as war simulations.

In today's era of technology, many things are wrapped in digital form to make it easy to provide information to the public. Many forms of information distribution can be done such as by websites, videos, images, print media, social media, and even applications. Currently, many applications have applied artificial intelligence, such as Augmented Reality technology on camera smartphones like face tracking on Instagram, Line, and Snapchat.
However, for application developers, it is not easy to create or search objects as a media marker, especially for the implementation of Markerless Augmented Reality where markers are objects that appear in everyday life.

Like in the Museum, many objects or photographs that are still originally black and white with texture, style and standard quality photographs on its time.

The amount of historical documentation still in the form of BNW photos can be used as a medium to introduce information/history on the object using Augmented Reality technology. A plug-in for Unity 3D to build Augmented Reality technology can use the Vuforia Software Development Kit (SDK).

The Vuforia SDK plugin cannot recognize the BNW image which was taken directly from the camera, so the developer has difficulty in identifying the pattern on the BNW image. This study was done in order for the marker to be detected by the camera, this study is entitled as "Marker Quality Improvement in Marker Augmented Reality Technology On Black and White Image (BNW) Using Regreyscale Method.

\section{MATERIAL AND METHOD}

\section{A. Augmented Reality}

Augmented Reality (AR) is a form of technology that can describe and combine the real world and virtual worlds created through computers, so the boundary between the two becomes very thin [1].

Augmented Reality (AR) is a variation of Virtual Environments (VE), or Virtual Reality as it is more commonly called. VE technologies completely immerse a user in a synthetic environment. While immersed, the user 
cannot see the real world around him. In contrast, AR allows the user to see the real world, with virtual objects superimposed upon or composited with the real world [2].

\section{B. Augmented Reality Method}

There are two primary types of AR implementations: Marker-based and Markerless [3]. In displaying AR, there are two ways that can be done such as Marker Augmented Reality (Marker Based Tracking) are using markers to display information, and another Markerless Augmented Reality which displays information without using markers such as Face Tracking, 3D Object Tracking, and Motion Tracking.

\section{Augmented Reality Applications}

The use of real-life Augmented Reality (AR) technology can be applied in advertisements, Computerized Tomography Scanner (CT Scan) or Magnetic Resonance Imaging (MRI) in the medical field, AR Game in entertainment and war simulation in the military [4]

\section{Grey-Scale Image}

In computing, a grayscale digital image is an image where the value of each pixel is a single sample. The image displayed in this type consists of grey, varying in black on the weakest intensity and white in the strong intensity. The grayscale image is different from the "black-and-white" image, which in the computer context; the black and white image is made up of only two colors: "black" and "white" only. In the grayscale image, the color varies between black and white, but the color variations are numerous. The grayscale image is often a calculation of the light intensity of each pixel in the single band electromagnetic spectrum [5].

Grayscale images are distinct from one-bit black-andwhite images, which in the context of computer imaging are images with only the two colors, black, and white (also called bi-level or binary images). Single bit black-and-white images are called binary images. Essentially, standard grayscale images are actually 8-bit black-and-white images such that for each pixel, there is a set of 28 permissible values. Due to this, grayscale images have many shades of grey in between [6]

Grayscales is a series of shades of grey without apparent color. The total absence of transmitted or reflected light, which is the darkest shade, black. The total reflection or transmission of light at all observable wavelengths, which is nothing but lightest possible shade i.e., white [7]

Grayscale conversion is also a vital part of image processing. RGB or color information has a 3 dimensional property which makes signal processing so much bulky and heavy to remove these drawbacks grey scale conversion is necessary [8].

\section{E. Vuforia SDK}

Vuforia is an Augmented Reality Software Development Kit (SDK) for mobile devices that enables the creation of Augmented Reality applications. It uses Computer Vision technology to recognize and track planar images (Image Targets) and simple 3D objects, such as boxes, in real-time [9].

Vuforia SDK is a software library written in the Java programming language for creating AR applications. It is distributed free for commercial and non-commercial applications. The following diagram provides an overview of the application development process with Vuforia platform[10].

Vuforia interacts with a mobile camera to be used as input devices, as an electronic eye that recognizes certain markers, so that the screen can display a combination of the real world and the world drawn by the application. In other words, Vuforia is the SDK for computer vision-based AR. Other AR application types are ArToolkit, OsgArt, and OpenCV.

Matlab is a numerical computing environment and programming language allowing easy matrix manipulation, plotting of functions and data, implementation of algorithms, the creation of user interfaces, and interfacing with programs in other languages. Although it is "numeric only" system, an optional toolbox interfaces with the Maple symbolic engine, allowing access to the computer algebra capabilities [11].

\section{F. Marker Quality}

One of the most frequently used approaches for image analysis in obtaining the necessary data are markers, providing Benchmarks and distinguishing internal marker elements according to which the objects can be identified and focused on [12].

Vuforia SDK using a natural feature to detect the fiducial marker. Fiducial marker tracking uses artificial markers which can be attached on the surface of an object to track [13].

Unlike a traditional marker/QR-code these images don't need special black and white regions or codes to be recognized because Vuforia uses algorithms to detect and track the features that are naturally found in the image itself [14].

Vuforia SDK recognizes the image target by comparing these natural features against a known target resource database.[15]. Once the Image Target is detected, the SDK will track the image as long as it is at least partially in the camera's field of view.

Table 1: Feature used on Vuforia SDK

\begin{tabular}{|l|l|}
\hline Shape Pixel & Feature Description \\
\hline & 4 angle patterns \\
\hline & Without angle \\
\hline
\end{tabular}

Marker quality can be measured by the stars acquired after uploading marker on the website developer.vuforia.com, the number of 1-2 stars means difficult to detect, 3 stars means sometimes can be detected and 4-5 stars are easily detected by the camera. The more patterns the more the star rating for the marker, which makes it easier for the camera to detect. The rating was based on image detail, contrast, and no pattern repetition. Feature on 
the Vuforia Software Development Kit (SDK) is a natural feature of an angle on each pixel of the image, positioned based on the camera's imaging range. The features used in the Vuforia SDK are shown in Table 1.

\section{G. Research Flow}

This study has two stages of research in which markers are tested by a process without Re-grayscale and markers with the Regrayscale process and then compare the marker quality that can be detected by the camera.

\section{H. Research Flow Diagram}

The diagram flow for this research is shown in Figure 1.

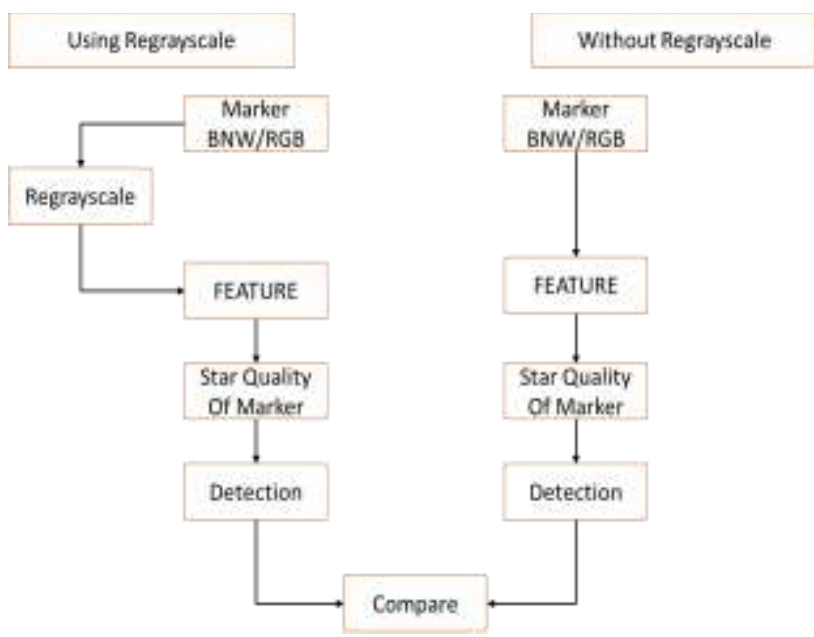

Figure 1: Research flow diagram.

\section{1) Objective}

This stage is intended to clarify the issues in order to make it easier for the next discussion. In addition, the formulation of the problem becomes very important because it is the basis and the ultimate goal of why this research is done. The problem raised in this research is whether BNW images taken directly from the camera can be used as markers on Augmented Reality technology.

\section{2) Data Source}

The source of data obtained from this research is BNW and RGB marker that comes from historical photographs taken from Siak Sri Indra Pura Palace, Riau, Indonesia which is captured directly using the smartphone camera.

\section{3) Technology and Tools Used}

The technology used for this application is Augmented Reality technology and the tool used as the Augmented Reality media is the marker, where the user will scan the market using the Smartphone camera to get information. It can be seen in Figure 2 is the workings of Augmented technology Reality (AR).

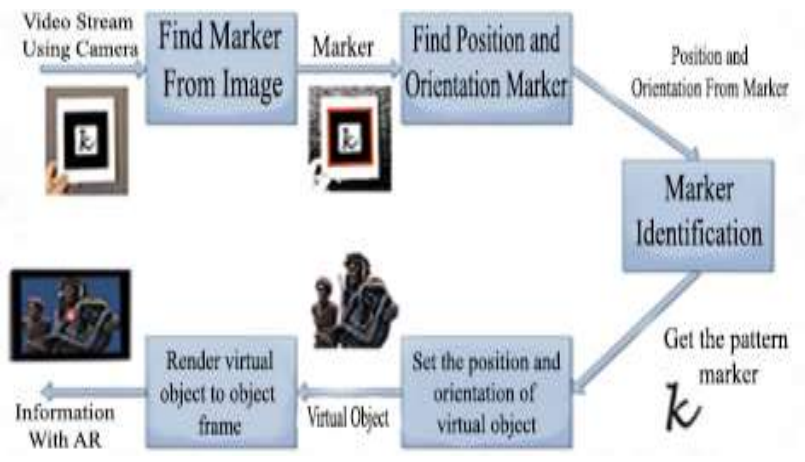

Figure 2: Augmented reality procedure.

The user performs a video stream using the camera, then searches for markers that have been marked as AR objects, then the system will automatically locate the marker orientation, position and identify the marker to get the marker pattern then adjust the position based on the orientation of the virtual object. After that render virtual objects into object frame to display user information.

\section{4) Markers Used}

A marker used is a Marker derived from historical photographs taken from the Palace of Siak Sri Indra Pura, Riau, Indonesia is taken directly using the camera. Markers can be seen in Table 2 .

Table 2: Marker used

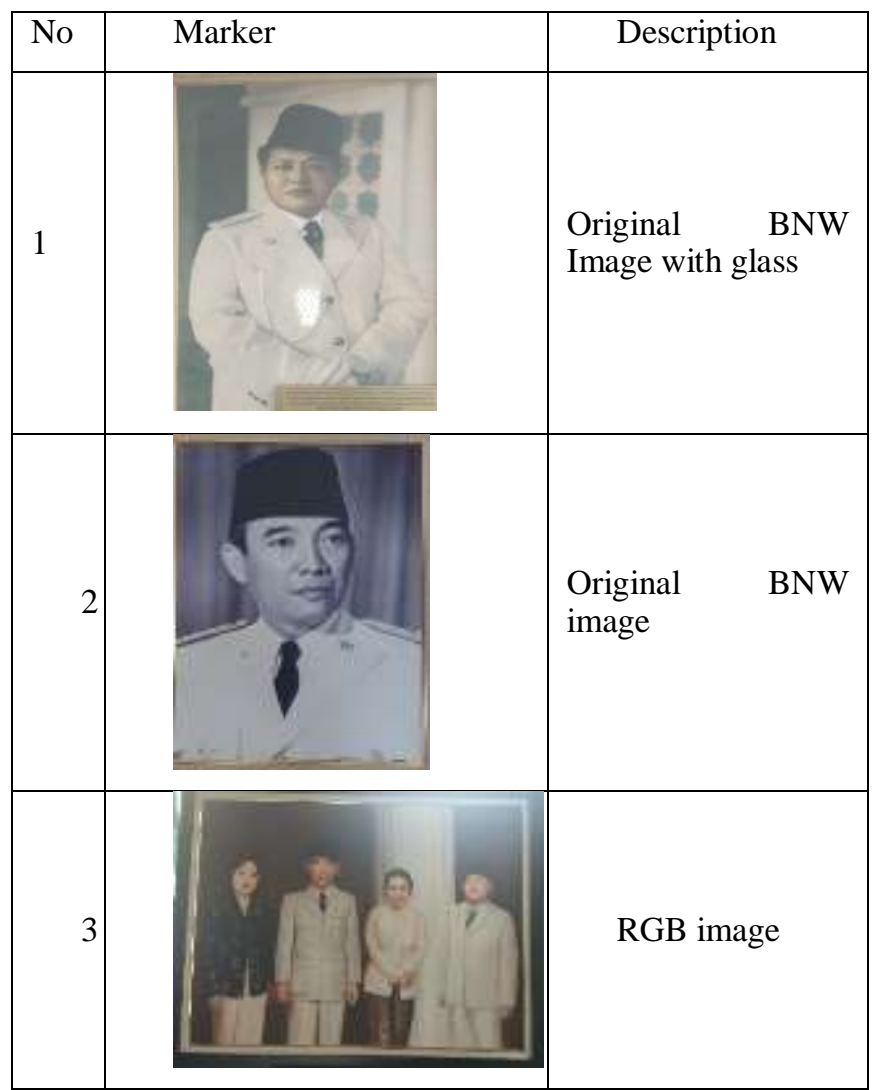




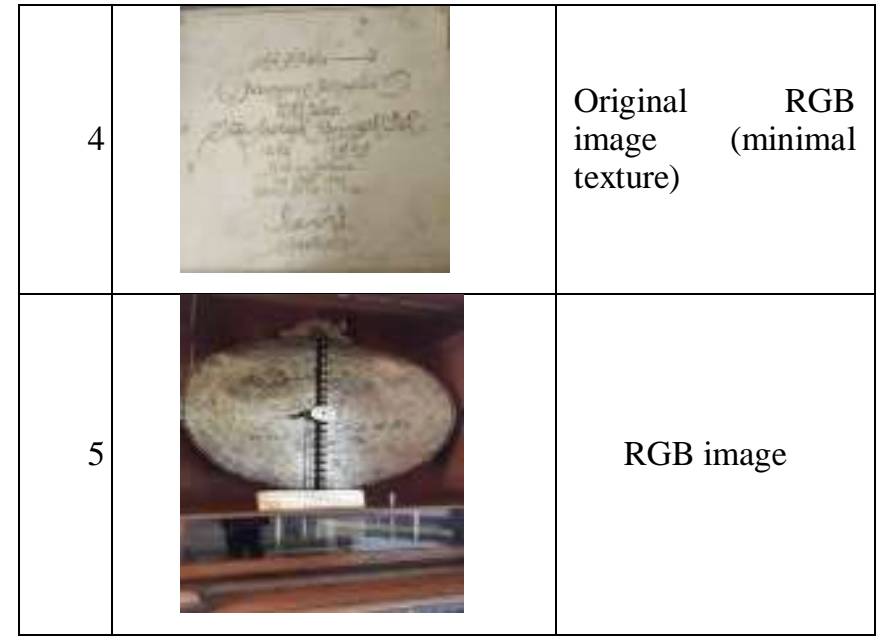

\section{5) Software}

For marker detection testing, required some software such as:

- Software Unity 3D as media to design Augmented Reality.

- Software Vuforia SDK as a plugin on the Unity 3D that serves as database markers and quality meter.

- Software Android SDK as a plugin in Unity 3D to develop Android applications.

6) Hardware

This test used the smartphone for image capture. The smartphone hardware specification used is shown in Table 3.

Table 3: Smartphone specification

\begin{tabular}{|c|l|}
\hline \multicolumn{2}{|c|}{ Smartphone Specification } \\
\hline Model & Samsung A8 + \\
\hline Processor & SoC Exynos 7885 Octa-core 2.2 Ghz \\
\hline RAM & $6.00 \mathrm{~GB}$ \\
\hline Operating System & Android Nougat 7.0 \\
\hline Camera & Dual camera 8MP and 16 MP \\
\hline
\end{tabular}

\section{RESUlts AND Discussion}

In the testing of marker quality on BNW image, the result was the improvement of marker quality which can be used as markers on AR technology.

\section{A. Upload Marker without Grayscales Process}

The marker is uploaded to the developer.vuforia.com website to determine the quality of the marker and see the features obtained as markers in Augmented Reality technology as shown in Table 4.

\section{B. Marker Result without Grayscales Process}

From Table 4 it can be concluded that marker quality in marker, no 1-4 has weak quality and cannot be used as markers on Augmented Reality technology either BNW marker or RGB marker, while marker, no 5 has 4 star quality and has many features so that marker can be used as markers on Augmented Reality technology.

Table 4: Marker used

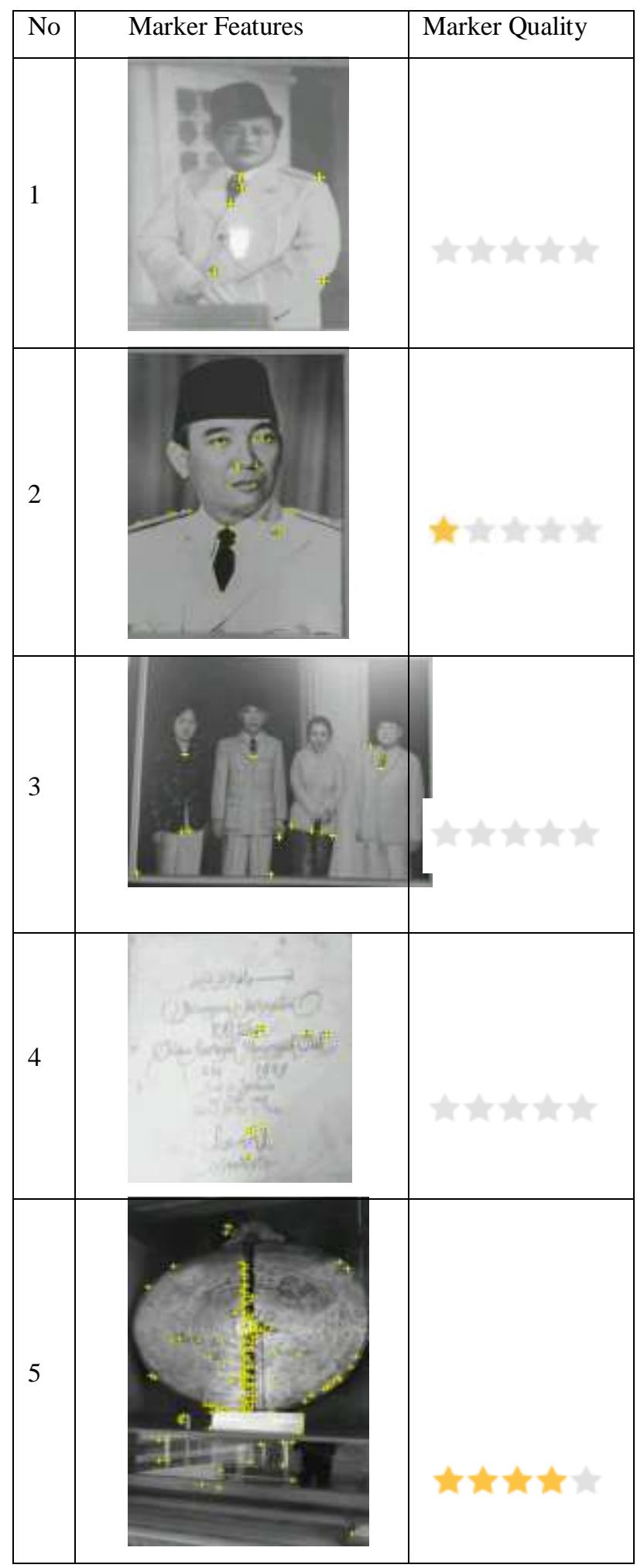

\section{Re-grayscale Process}

To improve marker quality was done by using Regrayscale process Matlab with syntax as in Figure 3 and the result in Figure 4. 


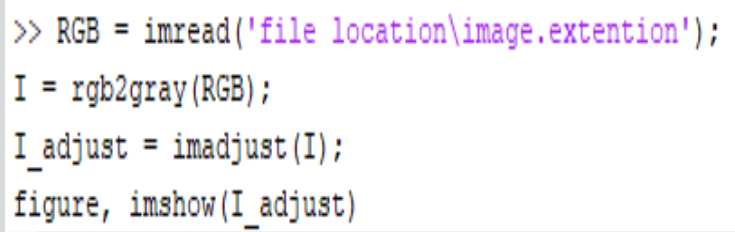

Figure 3: Syntax of re-grayscale.

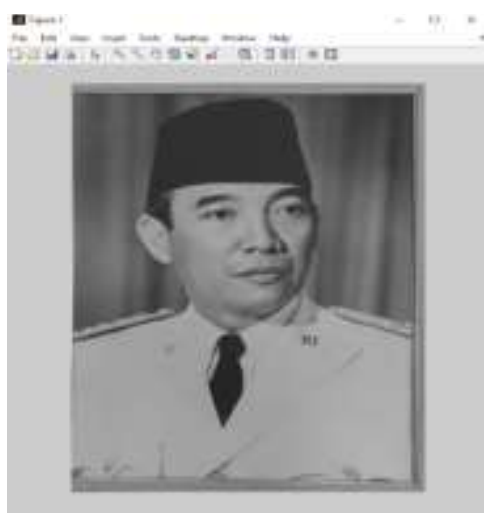

Figure 4: Re-grayscale using Matlab.

\section{Uploaded Marker After Re-grayscale}

After all, markers have been processed with Regrayscale using Matlab or another application that can change the image into RGB, marker then uploaded back to developer.vuforia.com to see the features and marker quality as shown in Table 5.

Table 4: Marker used

\begin{tabular}{|c|c|c|}
\hline No & Marker Features & Marker Quality \\
\hline 1 & & \\
& & \\
\hline & & \\
\hline
\end{tabular}

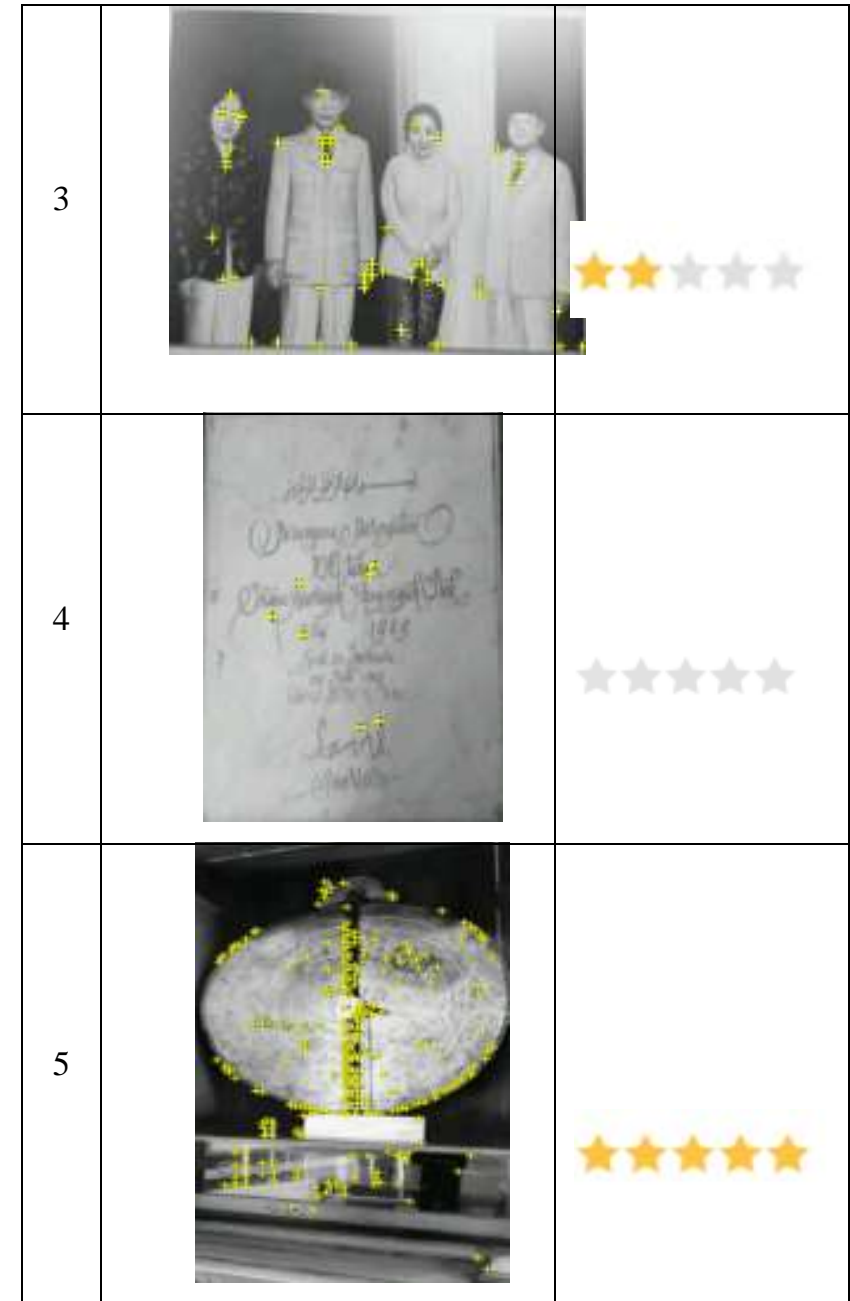

E. Marker Testing After Re-grayscale

Testing markers that have been re-grayscale were done on Android applications with AR technology embedded made by Unity 3D. The result of marker detection of the application is shown in Figure 5, Figure 6 and Table 6.

The figure label should put after the figure. No line spacing is needed between figure and label. However, ONE line space is needed between the figure with the previous paragraph and between the figure labels with the next paragraph. The Figure label should write in Times New Roman size 9. Example of the figure and its labeled as follow.

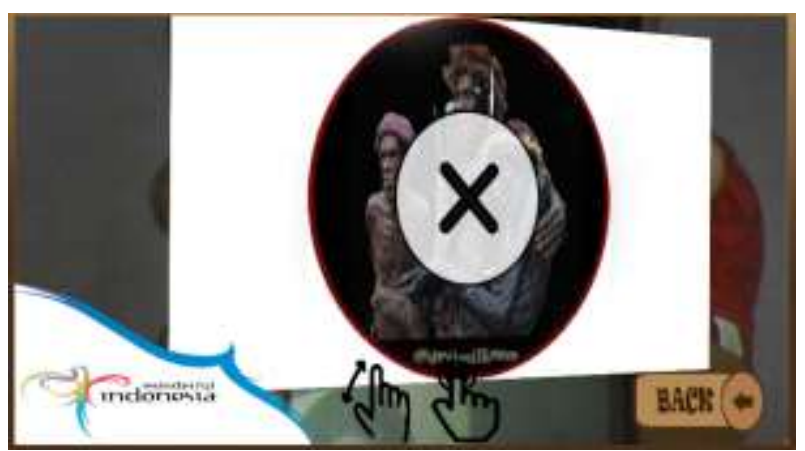

Figure 5: Marker test results detected by the camera. 


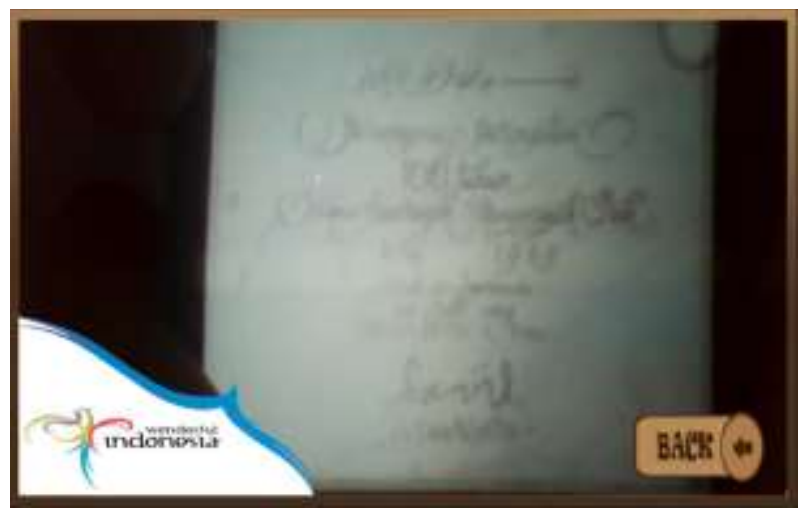

Figure 6: Marker test results detected by the camera.

Table 4: Marker used

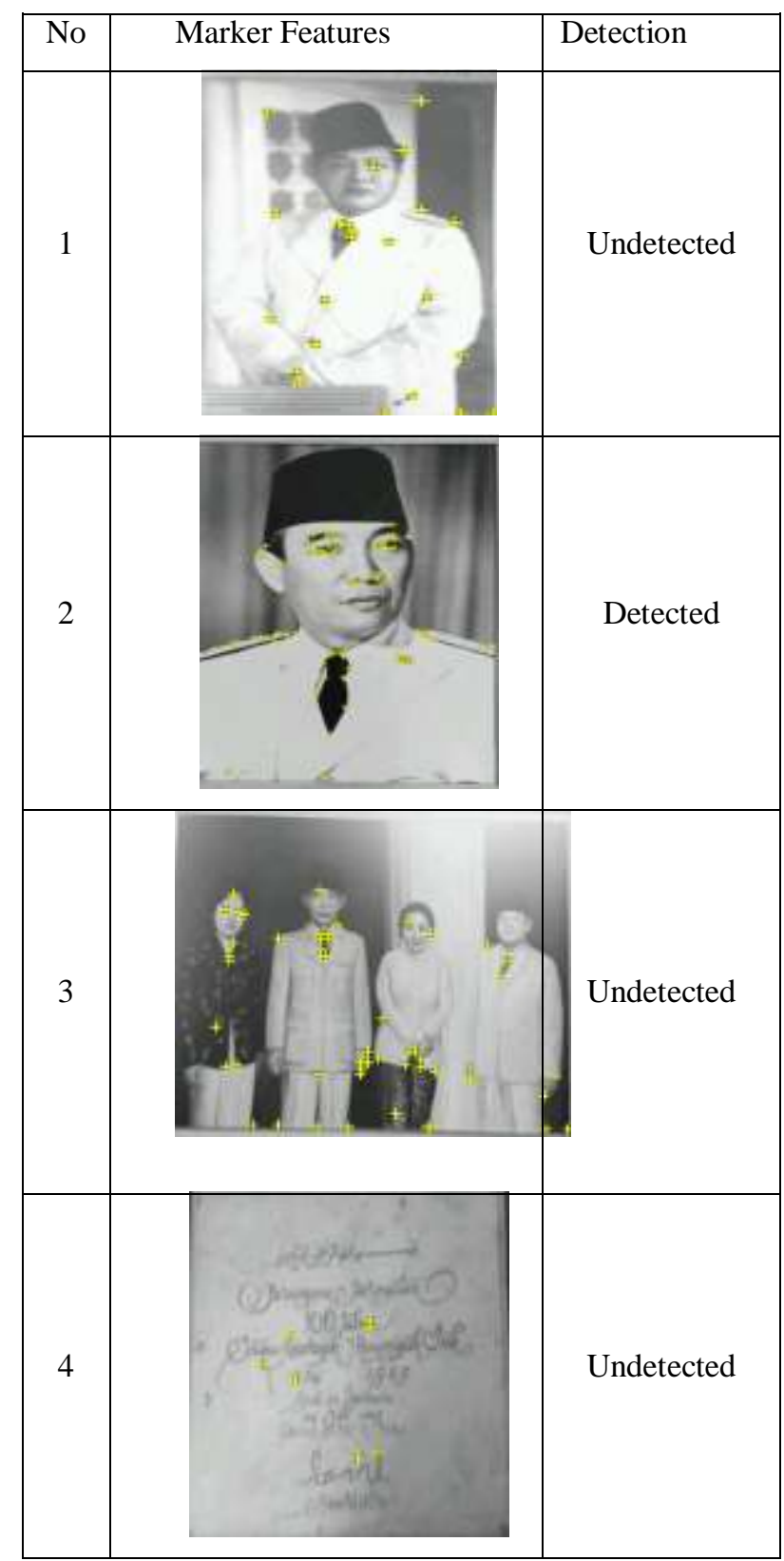

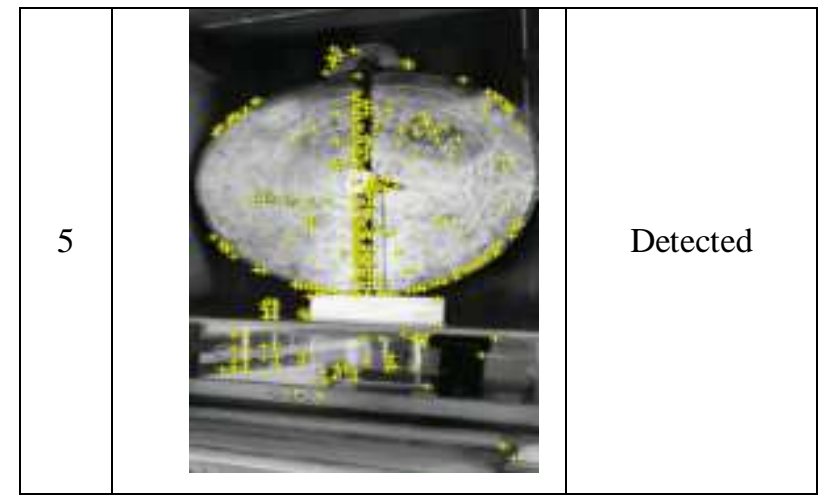

\section{CONCLUSION}

From the result and discussion about marker augmented reality, a conclusion can be drawn in marked improvement in image black and white (BNW) with Regrayscale method as follows:

- Marker 1 is the original BNW marker when the Regrayscale was done, and marker quality was increased by 2 stars, however the detail of Image texture was affected by the image capture process, which is interrupted by the glass covering the image.

- Marker 2 is a BNW marker that initially has one star after the Regrayscale process increased into 4 stars and was detected by AR-equipped camera.

- Marker 3 is an RGB marker, by doing Regrayscale the marker quality increases to 2 stars, but was undetectable due to lack of features in the image.

- Marker 4 is an RGB marker, after the Regrayscale process the number of stars did not increase, but the feature increased, it can be inferred also because of the lack of texture in the image.

- Marker 5 is an RGB marker with 4 stars, after the Regrayscale process, marker quality increased to 5 stars

\section{REFERENCES}

[1] P. A. Sudyatmika, P. N. Crisnapati, and I. G. M. Darmawiguna, "Pengembangan Aplikasi Augmented Reality Book Pengenalan Objek Wasita Taman Ujung Soekasada dan Taman Ar Tirta Gangga di Kabupaten Karangasem,” J. Pendidik. Teknol. dan Kejuru. 11.2, pp. 87-98, 2014.

[2] R. T. Azuma, "A Survey of Augmented Reality," vol. 4, no. August, pp. 355-385, 1997.

[3] Siltanen Sanni, Theory and applications of marker-based augmented reality. VTT Science 3, 2012.

[4] A. M. Lucio Tommaso De Paolis, Augmented and Virtual Reality. AVR: International Conference on Augmented and Virtual Reality, 2014

[5] H. Al Fatta, "Konversi Format Citra Rgb Ke Format Grayscale Menggunakan Visual Basic," vol. 2007, no. November, pp. 1-6, 2007.

[6] D. Biswas, A. Nag, S. Ghosh, A. Pal, S. Biswas, and S. Banerjee, "Novel Gray Scale Conversion Techniques Based On Pixel Depth" vol. 2, no. 6, pp. 118-121, 2011. 
[7] S. Jeyalaksshmi and S. Prasanna, "Measuring distinct regions of a grayscale image using pixel values," vol. 7, pp. 121-124, 2018.

[8] P. Kaler, "Study of Grayscale image in Image processing," no. November, pp. 309-311, 2016

[9] V. G. Karthiga, D. Beniel, A. K. M, and S. S. S, "Augmented Reality Game Development Using Unity \& Vuforia,” pp. 741-747, 2018.

[10] Kengne Paul Arol, 2014. Mobile Augmented Reality Supporting Marketing. Program in Business Information Technology, Autumn 2014

[11] J. Antoch, "Environment for statistical computing," vol. 2, pp. 113 122,2008
[12] D. Kurpytė and D. Navakauska, "An Efficiency Analysis of Augmented Reality Marker Recognition Algorithm,” 2014.

[13] R. Radkowski, “Object Tracking With a Range Camera for Augmented Reality Assembly Assistance," vol. 16, no. March, pp. 18, 2016.

[14] S. Nivedha and S. Hemalatha, “A Survey on Augmented Reality,” pp. 87-96, 2015.

[15] J. Bajana, D. Francia, A. Liverani, and M. Krajčovič, "Mobile tracking system and optical tracking integration for mobile mixed reality," vol. 53, no. 1, 2016 